\title{
A survey on recent results in Korovkin's approximation theory in modular spaces
}

\author{
CARlo BARDARO*, ANTONio Boccuto, AND Ilaria MANTEllini
}

\begin{abstract}
In this paper, we give a survey about recent versions of Korovkin-type theorems for modular function spaces, a class which includes $L^{p}$, Orlicz, Musielak-Orlicz spaces and many others. We consider various kinds of modular convergence, using certain summability processes, like triangular matrix statistical convergence, and filter convergence (which are generalizations of the statistical convergence). Finally, we consider an abstract axiomatic convergence which includes the previous ones and even almost convergence, which is not generated by any filter, as we show by an example.
\end{abstract}

Keywords: Korovkin's theorem, modular space, filter convergence, abstract convergence.

2020 Mathematics Subject Classification: 41A35, 47G10, 46E30, 40A35.

Dedicated to Professor Francesco Altomare, on occasion of his 70th birthday, with esteem and friendship.

\section{INTRODUCTION}

Korovkin's approximation theory is one of the main research topics of Professor Francesco Altomare. His famous monography ([3]), written in collaboration with Professor Michele Campiti, represents a fundamental reference point for any mathematicians who wish to study approximation theory by positive linear operators. Later on (see [2]), Altomare introduced a different approach to the theory developed in [3], obtaining general and unifying results.

The origin of this theory is the classical Bernstein proof of the Weierstrass theorem, where the author shows the uniform convergence of the Bernstein polynomials of a continuous function $f$ over the compact interval $[0,1]$ by stating it only for the test functions $\left\{1, x, x^{2}\right\}$, see [13]. This method was first generalized by H. Bohman ([18], who applied it to certain interpolating discrete operators acting on continuous functions over $[0,1])$ and then by P.P. Korovkin, for general positive linear operators [33] (see also the monograph [34]). In the meantime, also trigonometric versions of this basic result were obtained, by using the test functions $\{1, \cos x, \sin x\}$, (see e.g. [20]), and more generally functions which form a so-called Chebyshev system (that is a set of functions such that a linear combination of them has no more than two zeros on an interval, whenever its coefficients are not simultaneously null).

Several extensions in multivariate frame are also available in literature (see e.g. [12], [25], [43]).

The Korovkin theorem was successfully applied for stating uniform convergence for a very large class of integral or discrete positive operators, acting on continuous functions defined over compact intervals of the real line or also on compact sets in Euclidean spaces. Later,

Received: 03.10.2020; Accepted: 26.10.2020; Published Online: 01.03.2021

*Corresponding author: Carlo Bardaro; carlo.bardaro@unipg.it

DOI: $10.33205 / \mathrm{cma} .804697$ 
several extensions are obtained when the functions are defined on not necessarily compact intervals, using certain suitable weight functions, so obtaining weighted uniform convergence (see e.g. [29], [30]).

However, a unifying and general approach to Korovkin-type approximation was given in the quoted book [3] and in the extensive article [2], in which the authors expose the theory in the spaces $C(X)$, where $X$ is a locally compact topological space, together with its numerous applications.

Recently, versions of the Korovkin theorem were obtained in different functional spaces, namely $L^{p}$-spaces, or more general Lebesgue spaces (see [21], [26], [32], [42]), Orlicz-type spaces (see [37]). In this respect, many authors gave contributions to this theory and we refer to the exhaustive list of references of [2].

Our aim is to report a group of Korovkin-type theorems obtained by us in the abstract setting of the so-called modular spaces, a class of function spaces which includes $L^{p}$ spaces, Orlicz and Musielak-Orlicz spaces, weighted spaces, certain interpolation spaces, and many others. The theory of modular spaces was started by H. Nakano ([41]), but extensively studied by J. Musielak ([40]) and later on by W.M. Kozlowski ([36]). An abstract approach to the theory of approximation in modular spaces was given in [10]. The theory of Korovkin-type approximation in modular spaces enables one to obtain a unifying approach, which includes by a unique method, many previous results on the subject. Also, it is possible to obtain general results using a different notion of convergence, namely, filter-type convergence, including the case of the statistical convergence, relative uniform convergence with respect to scale functions, or an axiomatic abstract convergence, which includes filter convergence, triangular matrix statistical convergence and even almost convergence, which is not generated by any filter (see also [1], [4], [11], [15], [16], [17], [23], [24], [27], [28], [31], [39], [44]).

In Section 2, we introduce the modular function spaces for functions defined over nonempty open sets of an Hausdorff locally compact topological space. Then in Section 3, we report a Korovkin-type theorem for a family of positive linear operators acting on suitable subspaces of the modular spaces established in [8], [9], and we discuss some interesting examples. Then in Section 4, we report an extension of the previous results employing filter convergence, showing by examples that these new results are authentic extensions. Section 5 contains extensions of the Korovkin theorem with respect to generalized versions of statistical modular convergence, defined through certain summability processes involving regular matrices, and we give a comparison between this kind of convergence and filter convergence. In Section 6, we report some extensions of the Korovkin theorem to an abstract convergence introduced axiomatically, which includes the previous ones and other kinds of convergences not generated by any filter (see [5], [6], [17] and the references therein).

\section{MOdUlAR FUNCTION SPACES}

Let $G$ be a Hausdorff locally compact topological space endowed with a regular measure $\mu$ defined on the Borel sets of $G$.

We will denote by $L^{0}(G)$ the space of all real-valued Borel measurable functions $f: G \rightarrow \mathbb{R}$ provided with equality $\mu$-a.e. A functional $\varrho: L^{0}(G) \rightarrow \widetilde{\mathbb{R}_{0}^{+}}$is said to be a modular on $L^{0}(G)$ if

i) $\varrho[f]=0 \Leftrightarrow f=0$, a.e. in $\mathrm{G}$,

ii) $\varrho[-f]=\varrho[f]$, for every $f \in L^{0}(G)$,

iii) $\varrho[\alpha f+\beta g] \leq \varrho[f]+\varrho[g]$, for every $f, g \in L^{0}(G), \alpha, \beta \geq 0, \alpha+\beta=1$.

We will say that a modular $\varrho$ is Q-quasi convex if there is a constant $Q \geq 1$ such that

$$
\varrho[\alpha f+\beta g] \leq Q \alpha \varrho[Q f]+Q \beta \varrho[Q g],
$$


for every $f, g \in L^{0}(G), \alpha, \beta \geq 0, \alpha+\beta=1$. If $Q=1$, we will say that $\varrho$ is convex.

By means of the functional $\varrho$, we introduce the vector subspace of $L^{0}(G)$, denoted by $L^{\varrho}(G)$, defined by

$$
L^{\varrho}(G)=\left\{f \in L^{0}(G): \lim _{\lambda \rightarrow 0^{+}} \varrho[\lambda f]=0\right\} .
$$

The subspace $L^{\varrho}(G)$ is called the modular space generated by $\varrho$. It is easy to see that when $\varrho$ is $Q$-quasi-convex, we have the following characterization of the modular space $L^{\varrho}(G)$ :

$$
L^{\varrho}(G)=\left\{f \in L^{0}(G): \varrho[\lambda f]<+\infty \text { for some } \lambda>0\right\},
$$

see for example [40] and [10]. The subspace of $L^{\varrho}(G)$ defined by

$$
E^{\varrho}(G)=\left\{f \in L^{\varrho}(G): \varrho[\lambda f]<+\infty \text { for all } \lambda>0\right\}
$$

is called the space of the finite elements of $L^{\varrho}(G)$, see [40].

The following assumptions on modulars will be used:

a) $\varrho$ is monotone, i.e. for $f, g \in L^{0}(G)$ and $|f| \leq|g|$, then $\varrho[f] \leq \varrho[g]$.

b) $\varrho$ is finite, i.e. denoting by $e_{0}$ the function $e_{0}(t)=1$ for every $t \in G, e_{0} \in L \varrho(G)$.

c) $\varrho$ is absolutely finite, i.e. $\varrho$ is finite and for every $\varepsilon>0, \lambda>0$ there is $\delta>0$ such that $\varrho\left[\lambda \chi_{B}\right]<\varepsilon$ for any measurable subset $B \subset G$ with $\mu(B)<\delta$. Here, $\chi_{B}$ denotes the characteristic function of the set $B$.

d) $\varrho$ is strongly finite, i.e. $e_{0} \in E^{\varrho}(G)$.

e) $\varrho$ is absolutely continuous, i.e. there exists $\alpha>0$ such that for every $f \in L^{0}(G)$, with $\varrho[f]<+\infty$, the following condition is satisfied: for every $\varepsilon>0$ there is $\delta>0$ such that $\varrho\left[\alpha f \chi_{B}\right]<\varepsilon$, for every measurable subset $B \subset G$ with $\mu(B)<\delta$.

For the above notions, see [10], [40]. Note that, since $\mu(G)<+\infty$, if $\varrho$ is strongly finite and absolutely continuous, then it is also absolutely finite.

Classical examples of modular spaces are given by the Orlicz spaces generated by a $\varphi$-function $\varphi$ or, more generally, by any Musielak-Orlicz space generated by a $\varphi$-function $\varphi$ depending on a parameter, satisfying some growth condition with respect to the parameter (see [10], [36], [40] in some special cases). The modular functionals generating the above spaces satisfy all the previous assumptions.

We say that a sequence of functions $\left(f_{n}\right)_{n \in \mathbb{N}} \subset L^{\varrho}(G)$ is modularly convergent to a function $f \in L^{\varrho}(G)$, if there exists $\lambda>0$ such that

$$
\lim _{n \rightarrow+\infty} \varrho\left[\lambda\left(f_{n}-f\right)\right]=0 .
$$

This notion extends the norm-convergence in $L^{p}$-spaces. Moreover, it is weaker than the Fnorm-convergence induced by the Luxemburg F-norm generated by $\varrho$ and defined by

$$
\|f\|_{\rho} \equiv \inf \{u>0: \varrho[f / u] \leq u\} .
$$

We recall that a sequence of functions $\left(f_{n}\right)_{n \in \mathbb{N}}$ is F-norm-convergent (or strongly convergent) to $f$ if

$$
\lim _{n \rightarrow+\infty} \varrho\left[\lambda\left(f_{n}-f\right)\right]=0
$$

for every $\lambda>0$. The two notions of convergence are equivalent if and only if the modular satisfies the $\Delta_{2}$-condition, i.e. there exists a constant $M>0$ such that $\varrho[2 f] \leq M \varrho[f]$, for every $f \in L^{0}(G)$, see [40]. For example, this happens for all $L^{p}$-spaces and Orlicz spaces generated by $\varphi$-functions with the $\Delta_{2}$-regularity condition (see [10], [40]). The modular convergence induces a topology on $L^{\varrho}(G)$, called modular topology. Given a subset $\mathcal{B} \subset L^{\varrho}(G)$, we will denote by $\overline{\mathcal{B}}$ the closure of $\mathcal{B}$ with respect to the modular topology. Then $f \in \overline{\mathcal{B}}$ if there is a sequence $\left(f_{n}\right)_{n \in \mathbb{N}} \subset \mathcal{B}$ such that $f_{n}$ is modularly convergent to $f$. 
Let $A \subset G$ be an open set such that $\bar{A}$ is compact. We will denote by $L^{0}(A), L^{\varrho}(A), E^{\varrho}(A)$, the corresponding spaces. We denote by $C(A)$ the space of all continuous and bounded real functions defined on $A$ and by $C_{u}(A)$ the subset of $C(A)$ whose elements have a continuous extension to $\bar{A}$. It is easy to show that $C(A) \subset L^{\varrho}(A)$, whenever $\varrho$ is monotone and finite. Indeed, for $\lambda>0$ we have $\varrho[\lambda f] \leq \varrho\left[\lambda\|f\|_{\infty} e_{0}\right]$, and so, since $e_{0} \in L^{\varrho}(A)$, we have $\lim _{\lambda \rightarrow 0^{+}} \varrho[\lambda f]=0$, that is $f \in L^{\varrho}(A)$. Analogously, if $\varrho$ is monotone and strongly finite, then $C(A) \subset E^{\varrho}(A)$.

We will denote by $C_{c}(G)$ the subspace of $C(G)$ comprising all the functions with compact support on $G$. If $\varrho$ is monotone and finite, then $C_{c}(G) \subset L^{\varrho}(G)$, and if moreover $\varrho$ is strongly finite, $C_{c}(G) \subset E^{\varrho}(G)$.

We have the following (see [38]).

Proposition 1. Let $\varrho$ be a monotone, strongly finite and absolutely continuous modular on $L^{0}(G)$. Then $\overline{C_{c}(G)}=L^{\varrho}(G)$. Moreover, if $A \subset G$ is an open set such that $\bar{A}$ is compact, we have $\overline{C_{u}(A)}=L^{\varrho}(A)$.

\section{A KOROVKIN THEOREM FOR THE MODULAR CONVERGENCE}

Let $A \subset G$ be an open set with compact closure and let $e_{1}, \ldots e_{m}$ be $m$ functions in $C_{u}(A)$ such that the following property (P) holds: there exist continuous functions $a_{i} \in C_{u}(A), i=$ $1, \ldots m$ such that the function

$$
P_{s}(t)=\sum_{i=1}^{m} a_{i}(s) e_{i}(t), s, t \in \bar{A}
$$

is positive and is equal to zero if and only if $s=t$.

Let $\mathbf{T}=\left(T_{n}\right)_{n \in \mathbb{N}}$ be a family of positive linear operators $T_{n}: \mathcal{D} \rightarrow L^{0}(A)$, where $C_{u}(A) \subset$ $\mathcal{D} \subset L^{0}(A)$. Here, $\mathcal{D}$ is the domain of the operators $T_{n}$.

We will assume that the family $\left(T_{n}\right)_{n \in \mathbb{N}}$ satisfies the following property $(*)$ : there exist a subset $X_{\mathbf{T}} \subset \mathcal{D} \cap L^{\varrho}(A)$ with $C_{u}(A) \subset X_{\mathbf{T}}$ and a constant $R>0$ such that for every function $f \in X_{\mathbf{T}}$, we have $T_{n} f \in L^{\varrho}(A)$ and

$$
\limsup _{n \rightarrow+\infty} \varrho\left[\lambda\left(T_{n} f\right)\right] \leq R \varrho[\lambda f]
$$

for every $\lambda>0$.

Note that if $T_{n}: \mathcal{D} \rightarrow L^{0}(A)$ are equi-continuous operators in $L^{\varrho}(A)$, i.e.

$$
\varrho\left[\lambda T_{n} f\right] \leq R \varrho[\lambda f]
$$

for an absolute constant $R>0$, for every $\lambda>0$ and for every $f \in \mathcal{D} \cap L^{\varrho}(A)$, then clearly we can take $X_{\mathbf{T}}=L^{\varrho}(A) \cap \mathcal{D}$.

In what follows, we will assume that

$$
\lim _{n \rightarrow+\infty} T_{n} e_{i}=e_{i}, i=1, \ldots m \text { modularly in } L^{\varrho}(A) .
$$

A first result concerns the space $C_{u}(A)$ (see [8, Lemma 4]).

Theorem 1. Let $\varrho$ be a finite, monotone and $Q$-quasi-convex modular. Let the assumptions $(P)$ and (3.2) be satisfied. Then for every $f \in C_{u}(A)$, we have

$$
\lim _{n \rightarrow+\infty} T_{n} f=f \text { modularly in } L^{\varrho}(A) \text {. }
$$

Then, using Theorem 1 and the density result expressed by Proposition 1, the following result holds (see $[8$, Theorem 1$]$ ). 
Theorem 2. Let $\varrho$ be a monotone, strongly finite, absolutely continuous and Q-quasi-convex modular on $L^{0}(A)$. Let $\mathbf{T}=\left(T_{n}\right)_{n \in \mathbb{N}}$ be a sequence of positive linear operators satisfying property $(*)$. Let the assumption $(P)$ be satisfied. If

$$
\lim _{n \rightarrow+\infty} T_{n} e_{i}=e_{i} i=1, \ldots, m, \text { strongly in } L^{\varrho}(A),
$$

then $\lim _{n \rightarrow+\infty} T_{n} f=f$, modularly in $L^{\varrho}(A)$ for each $f \in L^{\varrho}(A) \cap \mathcal{D}$ such that $f-C_{u}(A) \subset X_{\mathbf{T}}$.

Theorem 2 can be applied to several positive linear operators in functional spaces. As example, it can be applied to positive operators of the form

$$
\left(S_{n} f\right)(x):=\sum_{k=0}^{r(n)} K_{n}\left(x, \nu_{n, k}\right) f\left(\nu_{n, k}\right) \quad(n \in \mathbb{N}, x \in A) \quad\left(f \in L^{\varrho}(A)\right) .
$$

Here, $A \subset \mathbb{R}^{N}$ is a bounded open set, $r(n)$ is an increasing sequence of natural numbers, and $\Gamma_{n}=\left(\nu_{n, k}\right)_{k=0,1, \ldots, r(n)} \subset A, \nu_{n, k}=\left(\nu_{n, k}^{1}, \ldots, \nu_{n, k}^{N}\right)$ is a sequence of points such that their union is dense in $A$.

In this and in the next example, we set $e_{0}(t)=1, e_{i}(t)=t_{i}, i=1,2, \ldots, N$ and $e_{N+1}(t)=|t|^{2}$, for every $t=\left(t_{1}, \ldots, t_{N}\right) \in A$. For details, see [8, Section 4$]$.

Another example is given by linear integral operators with positive kernel of Mellin-type. Let us consider $A=[0,1]^{N}$ and for any vectors $t=\left(t_{1}, \ldots, t_{N}\right), x=\left(x_{1}, \ldots, x_{N}\right) \in A$, we put $t x=\left(t_{1} x_{1}, \ldots, t_{N} x_{N}\right)$. Let $\left(K_{n}\right)_{n \in \mathbb{N}}$ be a sequence of kernel functions $K_{n}: A \rightarrow \mathbb{R}_{0}^{+}$such that

$$
\int_{A} K_{n}(t) d t=1 \text { and } \int_{A} \frac{K_{n}(t)}{t_{1} \cdots t_{N}} d t \leq W
$$

for every $n \in \mathbb{N}$, where $W$ is an absolute constant.

For any function $f \in L^{\varrho}(A)$, we define the positive linear operator

$$
\left(T_{n} f\right)(x)=\int_{A} K_{n}(t) f(t x) d t, x \in A .
$$

In this instance, we can show that $L^{\varrho}(A) \subset \mathcal{D}=\operatorname{Dom} \mathbf{T}=\bigcap_{n \in \mathbb{N}} \operatorname{Dom}_{n}$, where $\operatorname{Dom}_{n}$ is the subset of $L^{0}(A)$ on which $T_{n} f$ is well defined as a measurable function of $x \in A$. For details, see $[8$, Section 5].

\section{KOROVKIN'S THEOREM FOR FILTER MODULAR CONVERGENCE}

Here, we report extensions of Korovkin's theorem in modular spaces when the convergence is taken in a generalized form, namely involving filters. We begin with some properties of the filters of $\mathbb{N}$.

A nonempty family $\mathcal{F}$ of subsets on $\mathbb{N}$ is called a filter of $\mathbb{N}$ iff $\emptyset \notin \mathcal{F}, A \cap B \in \mathcal{F}$ whenever $A, B \in \mathcal{F}$ and for each $A \in \mathcal{F}$ and $A \subset B$ we have $B \in \mathcal{F}$. A sequence $\left(x_{n}\right)_{n} \subset \mathbb{R}$ is said to be $\mathcal{F}$-convergent to $x \in \mathbb{R}$ iff for every $\varepsilon>0$, the set $\left\{n \in \mathbb{N}:\left|x_{n}-x\right| \leq \varepsilon\right\}$ is an element of $\mathcal{F}$. We denote this by writing $x=(\mathcal{F}) \lim _{n} x_{n}$. We now introduce the filter versions of the limsup and liminf operations. Let $\underline{x}=\left(x_{n}\right)_{n}$ be a sequence in $\mathbb{R}$ and set

$$
A_{\underline{x}}=\left\{a \in \mathbb{R}:\left\{n \in \mathbb{N}: x_{n} \geq a\right\} \notin \mathcal{F}\right\}, B_{\underline{x}}=\left\{b \in \mathbb{R}:\left\{n \in \mathbb{N}: x_{n} \leq b\right\} \notin \mathcal{F}\right\} .
$$

The $\mathcal{F}$-limit superior and the $\mathcal{F}$-limit inferior of $\left(x_{n}\right)_{n}$ are defined by

$$
(\mathcal{F}) \limsup _{n} x_{n}=\left\{\begin{array}{ll}
\sup B_{\underline{x}} & \text { if } B_{\underline{x}} \neq \emptyset \\
-\infty & \text { if } B_{\underline{x}}=\emptyset
\end{array},(\mathcal{F}) \liminf _{n} x_{n}= \begin{cases}\inf A_{\underline{x}} & \text { if } A_{\underline{x}} \neq \emptyset \\
+\infty & \text { if } A_{\underline{x}}=\emptyset\end{cases}\right.
$$

respectively. For a related concept, see [22]. 
Among the examples of filters, we mention here the following two: the filter $\mathcal{F}_{\text {cofin }}$ of all subsets on $\mathbb{N}$ whose complement is finite, and the filter $\mathcal{F}_{d}$ associated with the statistical convergence, that is the set of all subsets of $\mathbb{N}$ whose asymptotic density is 1 (see e.g. [35]). In the first example, the notions of limsup and liminf coincide with the usual ones. The filter $\mathcal{F}_{\text {cofin }}$ is known as the Fréchet filter.

A filter $\mathcal{F}$ is said to be free if it contains the Fréchet filter. In the following, we assume that all the involved filters are free. We will use a uniformity $\mathcal{U} \subset 2^{G \times G}$ which generates the topology of the locally compact Hausdorff space $G$. Let $\mathcal{B}$ be the $\sigma$-algebra of all Borel subsets of $G$ and let $\mu$ be a $\sigma$-finite and regular measure on $\mathcal{B}$. Here, we denote by $C_{b}(G)$ the subspace of $C(G)$ comprising all the bounded functions on $G$. As before, $C_{c}(G)$ will denote the subspace of $C_{b}(G)$ containing all the functions with compact support on $G$. We define now the notion of modular convergence in a modular space $L^{\varrho}(G)$ in the context of filter convergence. A sequence $\left(f_{n}\right)_{n} \subset L^{\varrho}(G)$ is said to be $\mathcal{F}$-modularly convergent to $f \in L^{\varrho}(G)$ if there exists $\lambda>0$ such that

$$
(\mathcal{F}) \lim _{n} \varrho\left(\lambda\left(f_{n}-f\right)\right)=0 .
$$

Note that in case of the Fréchet filter, the filter modular convergence coincides with usual modular convergence. Also, with the same method, it is possible to introduce a notion of strong modular convergence in the context of filters.

In order to obtain an extension of the results of Section 3 , let $e_{i}, i=0,1, \ldots m$, be functions in $L^{\varrho}(G)$ such that (3.1) holds, in which we assume that it holds for every $s, t \in G$ and the following further conditions hold:

(P.1) $P_{s}(s)=0$ for all $s \in G$,

(P.2) for every $U \in \mathcal{U}$ there is $\delta>0$ such that for $s, t \in G$ such that $(s, t) \notin \mathcal{U}$ one has $P_{s}(t) \geq \delta$.

In [7, Example 4.1], there are described several examples of functions $P_{s}(t)$ satisfying the above assumptions. Moreover, we will modify slightly assumption $(*)$ simply replacing the space $C_{u}(A)$ by $C_{b}(G)$.

We have the following extension.

Theorem 3. Let $\varrho$ be a strongly finite, monotone and $Q$-quasi convex modular. Assume that the functions $e_{i}$ and $a_{i}, i=0,1, \ldots, m$, satisfy assumptions (P.1) and (P.2). Let $\left(T_{n}\right)_{n}$ be a sequence of positive linear operators satisfying the modified property $(*)$. If $T_{n} e_{i}$ is $\mathcal{F}$-modularly convergent to $e_{i}$ for $i=0, \ldots, m$ in $L^{\varrho}(G)$, then $T_{n} f$ converges $\mathcal{F}$-modularly to $f$ in $L^{\varrho}(G)$ for every $f \in C_{c}(G)$. If $T_{n} e_{i}$ is $\mathcal{F}$-strongly convergent to $e_{i}, i=0,1, \ldots, m$ in $L^{\varrho}(G)$, then $T_{n} f$ is $\mathcal{F}$-strongly convergent to $f \in L^{\varrho}(G)$ for every $f \in C_{c}(G)$.

The next theorem, which uses as a Lemma the previous theorem, extends also some previous Korovkin-type theorems in the setting of the statistical convergence, since the filter modular convergence is a generalization of the statistical convergence.

Theorem 4. Let $\varrho$ be a strongly finite, monotone, absolutely continuous and $Q$-quasi convex modular on $L^{0}(G)$. Let $\left(T_{n}\right)_{n}$ be a sequence of positive linear operators satisfying the modified property $(*)$. If $T_{n} e_{i}$ is $\mathcal{F}$-strongly convergent to $e_{i}, i=0,1, \ldots, m$, in $L^{\varrho}(G)$, then $T_{n} f$ is $\mathcal{F}$-modularly convergent to $f$ in $L^{\varrho}(G)$ for all $f \in L^{\varrho}(G) \cap \mathcal{D}$ such that $f-C_{b}(G) \subset X_{\mathbf{T}}$.

Further extensions of Theorem 4 can be also obtained for not necessarily positive linear operators, in case of functions defined over bounded intervals $I \subset \mathbb{R}$ with certain regularity assumptions (see [4], [7]). 


\section{DOUble SEQUENCES OF POSITIVE LINEAR OPERATORS AND KOROVKIN'S THEOREM WITH RESPECT TO A GENERALIZED VERSION OF STATISTICAL CONVERGENCE}

Here, we report a generalized version of the Korovkin theorem in case of double sequences of positive linear operators, employing a generalized concept of statistical convergence which involves regular matrices and their submatrices. This theory started in [5] in which a kind of "triangular matrix statistical convergence" was introduced for double sequences of positive linear operators in the space of continuous functions over compact subsets of $\mathbb{R}^{2}=\mathbb{R} \times \mathbb{R}$. This notion represents an extension of the known $A$-statistical convergence, defined through a regular matrix $A$, extensively studied in several previous papers (see e.g. [4], [24], [27], [39]).

For what concerns modular function spaces, in [6] we introduce a generalization of the triangular $A$-statistical convergence. In the present section, we describe this new method.

Let $A=\left(a_{i, j}\right)_{i, j}$ be a two-dimensional infinite matrix. Given a double sequence $x=\left(x_{i, j}\right)_{i, j}$ of real numbers, set

$$
(A x)_{i}:=\sum_{j=1}^{\infty} a_{i, j} x_{i, j},
$$

provided that the series is convergent. We say that $A$ is regular if it maps every convergent sequence into a convergent sequence with the same limit. We now recall the Silverman-Toeplitz conditions, which are a characterization of regular two-dimensional matrix transformations (see e.g. [4]). Here, $\Psi: \mathbb{N} \times \mathbb{N} \rightarrow \mathbb{R}$ is a fixed function.

(i) $\|A\|=\sup _{i \in \mathbb{N}} \sum_{j=1}^{\infty}\left|a_{i, j}\right|<\infty$,

(ii) $\lim _{i} a_{i, j}=0$ for each $j \in \mathbb{N}$,

(iii) $\lim _{i} \sum_{j=1}^{\infty} a_{i, j}=1$.

We say that $A$ is a summability matrix iff it satisfies the following conditions:

$j \in \mathbb{N}, \Psi(i, j) \geq 0$

(A2) $\lim _{i} \sum_{j \in \mathbb{N}, \Psi(i, j) \geq 0} a_{i, j}>0$,

(A3) $\lim _{i} a_{i, j}=0$ for every $j \in \mathbb{N}$

(see also [17]).

Let $A=\left(a_{i, j}\right)_{i, j}$ be a nonnegative regular matrix, $K \subset \mathbb{N}^{2}, K_{i}:=\{j \in \mathbb{N}:(i, j) \in K$, $\Psi(i, j) \geq 0\}$ and let $\left|K_{i}\right|$ denote the cardinality of $K_{i}$, for each $i \in \mathbb{N}$. The $\Psi$-A-density of $K$ is defined by

$$
\delta_{A}^{\Psi}(K):=\lim _{i} \sum_{j \in K_{i}} a_{i, j},
$$

provided that the limit on the right-hand side exists in $\mathbb{R}$.

It is not difficult to check that the $\Psi$ - $A$-density satisfies the following properties:

j) $\delta_{A}^{\Psi}\left(\mathbb{N}^{2}\right)=1$,

ji) if $H_{1} \subset H_{2}$, then $\delta_{A}^{\Psi}\left(H_{1}\right) \leq \delta_{A}^{\Psi}\left(H_{2}\right)$,

jij) $\delta_{A}^{\Psi}\left(\mathbb{N}^{2} \backslash K\right)=1-\delta_{A}^{\Psi}(K)$, provided that $K$ has a $\Psi$ - $A$ density.

Note that from $\mathrm{j}), \mathrm{jj}$ ) and $\mathrm{jj}$ ), it follows that the family

$$
\mathcal{F}_{A}^{\Psi}:=\left\{K \subset \mathbb{N}^{2}: \delta_{A}^{\Psi}\left(\mathbb{N}^{2} \backslash K\right)=0\right\}
$$


is a filter of $\mathbb{N}^{2}$ (Observe that the notion of (free) filter of $\mathbb{N}^{2}$ and the corresponding concept of filter convergence can be given analogously as those of filter of $\mathbb{N}$ and filter convergence presented in Section 4). In order to prove that $\mathcal{F}_{A}^{\Psi}$ is free, it is enough to see that, if $p, q \in \mathbb{N}$ and $K:=\left\{(i, j) \in \mathbb{N}^{2}: i \geq p, j \geq q\right\}$, then $\delta_{A}^{\Psi}\left(\mathbb{N}^{2} \backslash K\right)=0$. Indeed, we have $\mathbb{N}^{2} \backslash K \subset K^{(1)} \cup K^{(2)}$, where

$$
K^{(1)}=\bigcup_{j=1}^{q-1}(\mathbb{N} \times\{j\}), \quad K^{(2)}=\bigcup_{i=1}^{p-1}(\{i\} \times \mathbb{N}) .
$$

From $(A 3)$, it follows that

$$
\delta_{A}^{\Psi}\left(K^{(1)}\right)=\lim _{i} \sum_{j \in[1, q-1], \Psi(i, j)=0} a_{i, j} \leq \lim _{i} \sum_{j=1}^{q-1} a_{i, j}=0 .
$$

Furthermore, note that $\delta_{A}^{\Psi}\left(K^{(2)}\right)=0$, since $K_{i}^{(2)}=\emptyset$ for all $i \geq p$. Hence, $\delta_{A}^{\Psi}\left(\mathbb{N}^{2} \backslash K\right)=0$, that is the claim.

Let $A=\left(a_{i, j}\right)_{i, j}$ be a nonnegative regular summability matrix. The double sequence $x=$ $\left(x_{i, j}\right)_{i, j}$ is said to be $\Psi$-A-statistically convergent to $L$ if for any $\varepsilon>0$ we get

$$
\lim _{i} \sum_{j \in K_{i}(\varepsilon)} a_{i, j}=0
$$

where $K_{i}(\varepsilon)=\left\{j \in \mathbb{N}: \Psi(i, j) \geq 0,\left|x_{i, j}-L\right| \geq \varepsilon\right\}$, and we write $s t_{A}^{\Psi}$-lim $x_{i, j}=L$.

By $s t_{A}^{\Psi}$-limsup $x_{i, j}$ and $s t_{A}^{\Psi}$-liminf $x_{i, j}$, we denote the quantities

$$
\limsup _{i} \sum_{j \in K_{i}(\varepsilon)} a_{i, j} \quad \text { and } \liminf _{i} \sum_{j \in K_{i}(\varepsilon)} a_{i, j},
$$

respectively.

If we take $\Psi(i, j)=i-j$, we obtain the notion of triangular $A$-statistical convergence (see also [5]). Observe that, if $A=C_{1}$ is the Cesàro matrix, defined by setting

$$
a_{i, j}:=\left\{\begin{array}{cl}
\frac{1}{i} & \text { if } j \leq i, \\
0 & \text { otherwise, }
\end{array}\right.
$$

then the $\Psi$-A-statistical convergence can be considered as a generalized concept of the classical statistical convergence.

The $\Psi$-density $\delta^{\Psi}(K)$ is defined by

$$
\delta^{\Psi}(K)=\lim _{i} \frac{1}{i}\left|K_{i}\right| .
$$

The double sequence $x=\left(x_{i, j}\right)_{i, j}$ is said to be $\Psi$-statistically convergent to $L$ if for each $\varepsilon>0$ the set $K(\varepsilon):=\left\{(i, j) \in \mathbb{N}^{2}: \Psi(i, j) \geq 0,\left|x_{i, j}-L\right| \geq \varepsilon\right\}$ has triangular density zero, and we write $s t^{\Psi}-\lim _{i} x_{i, j}=L$.

We denote by $s t_{A}^{\Psi}$ the set of all $\Psi$-A-statistically convergent sequences.

Observe that, thanks to (5.4), filter convergence in $\mathbb{N}^{2}$ is weaker than $\Psi$ - $A$-statistical convergence, but in general these two convergences are not equal (see also [17, Section 4]). Indeed, we claim that there is some filter $\mathcal{F}$ of $\mathbb{N}^{2}$ such that, for each summability matrix $A$, there exists a set $K \in \mathcal{F} \backslash \mathcal{F}_{A}^{\Psi}$. Pick arbitrarily a summability matrix $A=\left(a_{i, j}\right)_{i, j}$. Thanks to $(A 1)$ and $(A 2)$, it is possible to find a real number $B_{0} \in(0,1]$ and an infinite subset $S \subset \mathbb{N}$ with 
$\sum_{j \in \mathbb{N}, \Psi(i, j) \geq 0} a_{i, j} \geq B_{0}$ for all $i \in S$ (see [17]). Let $S_{1}$ and $S_{2}$ be two disjoint infinite subsets of $S$, with $S=S_{1} \cup S_{2}$. Let $K:=\{(i, 1): i \in \mathbb{N}\} \cup\left\{(i, j): i \in S_{1}, \Psi(i, j) \geq 0\right\}$. Taking into account $(A 3)$, we get

$$
\begin{aligned}
0 & \leq \liminf _{i \in \mathbb{N} \backslash S_{1}} \sum_{j \in \mathbb{N}:(i, j) \in K, \Psi(i, j) \geq 0} a_{i, j} \leq \limsup _{i \in \mathbb{N} \backslash S_{1}} \sum_{j \in \mathbb{N}:(i, j) \in K, \Psi(i, j) \geq 0} a_{i, j} \\
& \leq \limsup _{i} a_{i, 1}=\lim _{i} a_{i, 1}=0 .
\end{aligned}
$$

Thus, all inequalities in (5.5) are equalities, and in particular it is

$$
\lim _{i \in \mathbb{N} \backslash S_{1}} \sum_{j \in \mathbb{N}:(i, j) \in K, \Psi(i, j) \geq 0} a_{i, j}=0 .
$$

Since $\limsup _{i \in S_{1}} \sum_{j \in \mathbb{N}:(i, j) \in K, \Psi(i, j) \geq 0} a_{i, j} \geq B_{0}$, from this and (5.6), it follows that $\mathbb{N}^{2} \backslash K \notin \mathcal{F}_{A}^{\Psi}$. From this and $(A 2)$, it follows that $K \notin \mathcal{F}_{A}^{\Psi}$. So, any ultrafilter $\mathcal{F}$ of $\mathbb{N}$ contains at least a set not belonging to $\mathcal{F}_{A}^{\Psi}$, since it contains either $K$ or $\mathbb{N}^{2} \backslash K$. This gets the claim.

We now define the modular and strong convergences in the context of the $\Psi$ - $A$-statistical convergence. In the following, $G$ is a locally compact Hausdorff space satisfying the assumptions of Section 4, with the uniform structure $\mathcal{U} \subset 2^{G \times G}$.

We say that a double sequence $\left(f_{i, j}\right)_{i, j}$ of functions in $L^{\varrho}(G)$ is $s t_{A}^{\Psi}$-modularly convergent to $f \in L^{\varrho}(G)$ if there is a $\lambda>0$ with

$$
s t_{A}^{\Psi}-\lim _{i} \varrho\left[\lambda\left(f_{i, j}-f\right)\right]=0 .
$$

A double sequence $\left(f_{i, j}\right)_{i, j}$ in $L^{\varrho}(G)$ is $s t_{A}^{\Psi}$-strongly convergent to $f \in L^{\varrho}(G)$ if

$$
s t_{A}^{\Psi}-\lim _{i} \varrho\left[\lambda\left(f_{i, j}-f\right)\right]=0
$$

for every $\lambda>0$.

For $\Psi(i, j)=i-j$, we obtain the corresponding notions for the "triangular" modular and strong convergences.

Let $T$ be a double sequence of linear operators $T_{i, j}: \mathcal{D} \rightarrow L^{0}(G), i, j \in \mathbb{N}$, with $C_{b}(G) \subset \mathcal{D} \subset$ $L^{0}(G)$. Here, the set $\mathcal{D}$ is the domain of the operators $T_{i, j}$.

We introduce now the following extension of the property $(*)$ for double sequences of positive linear operators. We say that the double sequence $T$, together with the modular $\varrho$, satisfies the property (*) if there exist a subset $X_{T} \subset \mathcal{D} \cap L^{\varrho}(G)$ with $C_{b}(G) \subset X_{T}$ and a positive real constant $N$ with $T_{i, j} f \in L^{\varrho}(G)$ for any $f \in X_{T}$ and $i, j \in \mathbb{N}$, and $s t_{A}^{T}$ - $\limsup _{i} \varrho\left[\tau\left(T_{i, j} f\right)\right] \leq N \varrho[\tau f]$ for every $f \in X_{T}$ and $\tau>0$.

The first Korovkin-type theorem in the present setting is the following (see [6]).

Theorem 5. Let $\varrho$ be a strongly finite, monotone and $Q$-quasi convex modular. Assume that $e_{r}$ and $a_{r}$, $r=0, \ldots, m$, satisfy (P1) and (P2). Let $T_{i, j}, i, j \in \mathbb{N}$, be a double sequence of positive linear operators with property $(*)$. If $\left(T_{i, j} e_{r}\right)_{i, j}$ is st $A_{A}^{\Psi}$-modularly convergent to $e_{r}$ in $L^{\varrho}(G)$ for each $r=0, \ldots, m$, then $\left(T_{i, j} f\right)_{i, j}$ is st ${ }_{A}^{\Psi}$-modularly convergent to $f$ in $L^{\varrho}(G)$ for every $f \in C_{c}(G)$.

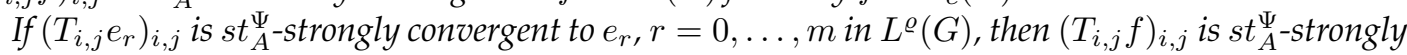
convergent to $f$ in $L^{\varrho}(G)$ for every $f \in C_{c}(G)$.

Employing Theorem 5, we can prove the following general Korovkin-type theorem in modular spaces (see [6]). 
Theorem 6. Let $\varrho$ be a monotone, strongly finite, absolutely continuous and $Q$-quasi convex modular on $L^{0}(G)$, and $T_{i, j}, i, j \in \mathbb{N}$ be a double sequence of positive linear operators fulfilling $(*)$. If $\left(T_{i, j} e_{r}\right)_{i, j}$ is st $A_{A}^{\Psi}$-strongly convergent to $e_{r}, r=0, \ldots, m$ in $L^{\varrho}(G)$, then $\left(T_{i, j} e_{r}\right)_{i, j}$ is st $t_{A}^{\Psi}$-modularly convergent to $f$ in $L^{\varrho}(G)$ for every $f \in L^{\varrho}(G) \cap \mathcal{D}$ with $f-C_{b}(G) \subset X_{T}$, where $\mathcal{D}$ and $X_{T}$ are as before.

The theory developed in this section can be applied to two-dimensional Mellin-type integral operators for functions defined in compact intervals of $\mathbb{R}^{2}$. As example, let $L^{\varphi}(G)$ be an Orlicz space generated by the convex $\varphi$-function $\varphi$ and $G=[0,1]^{2}$.

For every $\left(x_{1}, x_{2}\right) \in[0,1]^{2}$, let $e_{0}\left(x_{1}, x_{2}\right)=a_{3}\left(x_{1}, x_{2}\right)=1, e_{1}\left(x_{1}, x_{2}\right)=x_{1}, e_{2}\left(x_{1}, x_{2}\right)=x_{2}$, $e_{3}\left(x_{1}, x_{2}\right)=a_{0}\left(x_{1}, x_{2}\right)=x_{1}^{2}+x_{2}^{2}, a_{1}\left(x_{1}, x_{2}\right)=-2 x_{1}, a_{2}\left(x_{1}, x_{2}\right)=-2 x_{2}$. For each $i, j \in \mathbb{N}$, set $A_{i, j}=\left[\frac{1}{i}, 1\right] \times\left[\frac{1}{j}, 1\right]$,

$$
c_{i, j}=\int_{0}^{1} \int_{0}^{1} t_{1} t_{2}\left(t_{1}^{2}+t_{2}^{2}\right)^{i+j} \chi_{A_{i, j}}\left(t_{1}, t_{2}\right) d t_{1} d t_{2}, \quad d_{i, j}=\frac{1}{c_{i, j}} .
$$

For every $i, j \in \mathbb{N}, t_{1}, t_{2} \in[0,1]$, define $K_{i, j}\left(t_{1}, t_{2}\right)=d_{i, j} t_{1} t_{2}\left(t_{1}^{2}+t_{2}^{2}\right)^{i+j} \chi_{A_{i, j}}\left(t_{1}, t_{2}\right)$. Moreover, let $C_{1}$ be the Cesàro matrix. Finally, let us consider the double sequences of operators defined by

$$
\left(M_{i, j} f\right)\left(x_{1}, x_{2}\right)=\int_{0}^{1} \int_{0}^{1} K_{i, j}\left(t_{1}, t_{2}\right) f\left(t_{1} x_{1}, t_{2} x_{2}\right) d t_{1} d t_{2}, \quad f \in C\left([0,1]^{2}\right), \quad i, j \in \mathbb{N} .
$$

It is proved that the operators $M_{i, j}$ satisfy all the assumptions of Theorem 5 .

\section{KOROVKIN THEOREMS WITH RESPECT TO ABSTRACT CONVERGENCE}

In this section, we extend the results of the previous sections to abstract convergences, including filter and triangular matrix statistical convergences. In [17], Theorems 3, 4, 5 and 6 were generalized by considering an axiomatic abstract convergence, defined as follows (see also [14]).

Let $\mathcal{T}$ be the set of all real-valued sequences $\left(x_{n}\right)_{n}$. A convergence is a pair $(\mathcal{S}, \ell)$, where $\mathcal{S}$ is a linear subspace of $\mathcal{T}$ and $\ell: \mathcal{S} \rightarrow \mathbb{R}$ is a function, satisfying the following axioms.

(a) $\ell\left(\left(a_{1} x_{n}+a_{2} y_{n}\right)_{n}\right)=a_{1} \ell\left(\left(x_{n}\right)_{n}\right)+a_{2} \ell\left(\left(y_{n}\right)_{n}\right)$ for every pair of sequences $\left(x_{n}\right)_{n},\left(y_{n}\right)_{n} \in$ $\mathcal{S}$ and for each $a_{1}, a_{2} \in \mathbb{R}$ (linearity).

(b) If $\left(x_{n}\right)_{n},\left(y_{n}\right)_{n} \in \mathcal{S}$ and $x_{n} \leq y_{n}$ definitely, then $\ell\left(\left(x_{n}\right)_{n}\right) \leq \ell\left(\left(y_{n}\right)_{n}\right)$ (monotonicity).

(c) If $\left(x_{n}\right)_{n}$ satisfies $x_{n}=l$ definitely, then $\left(x_{n}\right)_{n} \in \mathcal{S}$ and $\ell\left(\left(x_{n}\right)_{n}\right)=l$.

(d) If $\left(x_{n}\right)_{n} \in \mathcal{S}$, then $\left(\left|x_{n}\right|\right)_{n} \in \mathcal{S}$ and $\ell\left(\left(\left|x_{n}\right|\right)_{n}\right)=\left|\ell\left(\left(x_{n}\right)_{n}\right)\right|$.

(e) Given three sequences $\left(x_{n}\right)_{n},\left(y_{n}\right)_{n},\left(z_{n}\right)_{n}$, satisfying $\left(x_{n}\right)_{n},\left(z_{n}\right)_{n} \in \mathcal{S}, \ell\left(\left(x_{n}\right)_{n}\right)=$ $\ell\left(\left(z_{n}\right)_{n}\right)$ and $x_{n} \leq y_{n} \leq z_{n}$ definitely, then $\left(y_{n}\right)_{n} \in \mathcal{S}$.

Note that $\mathcal{S}$ is the space of all convergent sequences, and $\ell$ is the "limit" according to this approach.

We now give the axiomatic definition of the operators "limit superior" and "limit inferior" associated with a convergence $(\mathcal{S}, \ell)$.

Let $\mathcal{T}, \mathcal{S}$ be as above. We define two functions $\bar{\ell}, \underline{\ell}: \mathcal{T} \rightarrow \widetilde{\mathbb{R}}$, satisfying the following axioms:

(f) If $\left(x_{n}\right)_{n},\left(y_{n}\right)_{n} \in \mathcal{T}$, then $\underline{\ell}\left(\left(x_{n}\right)_{n}\right) \leq \bar{\ell}\left(\left(x_{n}\right)_{n}\right)$ and $\bar{\ell}\left(\left(x_{n}\right)_{n}\right)=-\underline{\ell}\left(\left(-x_{n}\right)_{n}\right)$.

(g) If $\left(x_{n}\right)_{n} \in \mathcal{T}$, then

(i) $\bar{\ell}\left(\left(x_{n}+y_{n}\right)_{n}\right) \leq \bar{\ell}\left(\left(x_{n}\right)_{n}\right)+\bar{\ell}\left(\left(y_{n}\right)_{n}\right)$ (subadditivity);

(ii) $\underline{\ell}\left(\left(x_{n}+y_{n}\right)_{n}\right) \geq \underline{\ell}\left(\left(x_{n}\right)_{n}\right)+\underline{\ell}\left(\left(y_{n}\right)_{n}\right)$ (superadditivity).

(h) If $\left(x_{n}\right)_{n},\left(y_{n}\right)_{n} \in \mathcal{T}$ and $x_{n} \leq y_{n}$ definitely, then $\bar{\ell}\left(\left(x_{n}\right)_{n}\right) \leq \bar{\ell}\left(\left(y_{n}\right)_{n}\right)$ and $\underline{\ell}\left(\left(x_{n}\right)_{n}\right) \leq$ $\underline{\ell}\left(\left(y_{n}\right)_{n}\right)$ (monotonicity). 
(j) A sequence $\left(x_{n}\right)_{n} \in \mathcal{T}$ belongs to $\mathcal{S}$ if and only if $\bar{\ell}\left(\left(x_{n}\right)_{n}\right)=\underline{\ell}\left(\left(x_{n}\right)_{n}\right)$.

It is not difficult to check that the $\mathcal{F}$-limit superior and the $\mathcal{F}$-limit inferior defined in (4.3) fulfill the above axioms (f)-(j) (see also [22, Theorems 3 and 4]).

Now, we define the modular and strong convergences in this setting.

A sequence $\left(f_{n}\right)_{n}$ of functions in $L^{\rho}(G)$ is $(\ell)$-modularly convergent to $f \in L^{\rho}(G)$ if there is a positive real number $\lambda$ such that

$$
(\ell) \lim _{n} \rho\left[\lambda\left(f_{n}-f\right)\right]=0 .
$$

A sequence $\left(f_{n}\right)_{n}$ in $L^{\rho}(G)$ is $(\ell)$-strongly convergent to $f \in L^{\rho}(G)$ if

$$
(\ell) \lim _{n} \rho\left[\lambda\left(f_{n}-f\right)\right]=0 \text { for all } \lambda>0 .
$$

The Korovkin-type theorems obtained in the context of this abstract axiomatic convergence are the following.

Theorem 7. ([17, Theorem 3.2]) Let $\rho$ be a strongly finite, monotone and Q-quasi convex modular. Assume that $e_{r}$ and $a_{r}, r=0, \ldots, m$, satisfy (P1) and (P2). Let $\left(T_{n}\right)_{n}$ be a sequence of positive linear operators satisfying property $(*)$. If $\left(T_{n} e_{r}\right)_{n}$ is $(\ell)$-modularly convergent to $e_{r}$ in $L^{\rho}(G)$ for each $r=0, \ldots, m$, then $\left(T_{n} f\right)_{n}$ is $(\ell)$-modularly convergent to $f$ in $L^{\rho}(G)$ for all $f \in C_{c}(G)$.

If $\left(T_{n} e_{r}\right)_{n}$ is $(\ell)$-strongly convergent to $e_{r}, r=0, \ldots, m$ in $L^{\rho}(G)$, then $\left(T_{n} f\right)_{n}$ is $(\ell)$-strongly convergent to $f$ in $L^{\rho}(G)$ for every $f \in C_{c}(G)$.

Theorem 8. ([17, Theorem 3.3]) Let $\rho$ be a monotone, strongly finite, absolutely continuous and $Q-$ quasi convex modular on $L^{0}(G)$, and $\left(T_{n}\right)_{n}$ be a sequence of positive linear operators satisfying $(*)$. If $\left(T_{n} e_{r}\right)_{n}$ is $(\ell)$-strongly convergent to $e_{r}, r=0, \ldots, m$ in $L^{\rho}(G)$, then $\left(T_{n} e_{r}\right)_{n}$ is $(\ell)$-modularly convergent to $f$ in $L^{\rho}(G)$ for every $f \in L^{\rho}(G) \cap \mathcal{D}$ with $f-C_{b}(G) \subset X_{T}$, where $\mathcal{D}$ and $X_{T}$ are as above.

Now, we give an example of convergence, which satisfies axioms (a)-(j) introduced in this section, but is not generated by any (free) filter (see also [7, Section 6]).

A sequence $\left(x_{n}\right)_{n}$ in $\mathbb{R}$ is almost convergent to $x_{0} \in \mathbb{R}$ (shortly, $(A) \lim _{n} x_{n}=x_{0}$ ) if

$$
\lim _{n} \frac{x_{m+1}+x_{m+2}+\ldots+x_{m+n}}{n}=x_{0}
$$

uniformly with respect to $m \in \mathbb{N}$. It is not difficult to check that almost convergence satisfies axioms (a)-(j).

Let $\mathcal{F}$ be any fixed free filter of $\mathbb{N}$. A function $f: \mathbb{R} \rightarrow \mathbb{R}$ is $\mathcal{F}$-continuous at $x_{0} \in \mathbb{R}$ if $(\mathcal{F}) \lim _{n} f\left(x_{n}\right)=f\left(x_{0}\right)$ whenever $(\mathcal{F}) \lim _{n} x_{n}=x_{0}$, and is $A$-continuous at $x_{0} \in \mathbb{R}$ if $(A) \lim _{n} f\left(x_{n}\right)=$ $f\left(x_{0}\right)$ whenever $(A) \lim _{n} x_{n}=x_{0}$. In [35, Proposition 3.3] it is shown that $\mathcal{F}$-continuity is equivalent to usual continuity, while in [19, Theorem 1] it is proved that every $(A)$-continuous function at any fixed point $x_{0}$ is linear. Thus, the concepts of $(A)$ - and $\mathcal{F}$-continuity do not coincide, and therefore almost convergence is not generated by any free filter.

As a final remark, note that in [6, Section 4] and [17, Theorems 3.4 and 3.5] some results about the rate of modular convergence were also stated, using suitable moduli of continuity, when $G$ is a metric space $(G, d)$ satisfying a suitable assumption, which is naturally verified in every Euclidean space. Following an approach already used in [7], it is also possible to obtain a version of the previous theorems for not necessarily positive linear operators, in certain particular situations (see [6, Theorem 12] and [17, Theorem 4.3]). 


\section{REFERENCES}

[1] O. Agratini: On statistical approximation in spaces of continuous functions, Positivity, 13 (4) (2009), 735-743.

[2] F. Altomare: Korovkin-type theorems and approximation by positive linear operators, Surv. Approx. Theory, 5 (2010), 92-164.

[3] F. Altomare, M. Campiti: Korovkin-type approximation theory and its applications, Walter de Gruyter, Berlin, New York, (1994).

[4] G. A. Anastassiou, O. Duman: Towards Intelligent Modeling: Statistical Approximation Theory, Intelligent System Reference Library 14, Springer-Verlag, Berlin, Heidelberg, New York, (2011).

[5] C. Bardaro, A. Boccuto, K. Demirci, I. Mantellini and S. Orhan: Triangular A-statistical approximation by double sequences of positive linear operators, Results. Math., 68 (2015), 271-291.

[6] C. Bardaro, A. Boccuto, K. Demirci, I. Mantellini and S. Orhan: Korovkin-type theorems for modular $\Psi$-A-statistical convergence, J. Function Spaces, 2015, Article ID 160401.

[7] C. Bardaro, A. Boccuto, X. Dimitriou and I. Mantellini: Abstract Korovkin-type theorems in modular spaces and applications, Cent. Eur. J. Math., 11 (10) (2013), 1774-1784.

[8] C. Bardaro, I. Mantellini: Korovkin theorem in modular spaces, Comment. Math., 47 (2) (2007), 239-253.

[9] C. Bardaro, I. Mantellini: A Korovkin theorem in multivariate modular spaces, J. Function Spaces Appl., 7 (2) (2009), 105-120.

[10] C. Bardaro, J. Musielak and G. Vinti: Nonlinear integral operators and applications, De Gruyter Series in Nonlinear Analysis and Appl., Berlin, Vol. 9, (2003).

[11] C. Belen, M. Yildirim: Statistical approximation in multivariate modular function spaces, Comment. Math., 51 (1) (2011), 39-53.

[12] H. Berens, G. G. Lorentz: Theorems of Korovkin type for positive linear operators on Banach lattices, in: Approximation Theory (Proc. Internat. Sympos. Univ. Texas,Austin,Texas (1973), 1-30; Academic Press, New York, (1973).

[13] S. N. Bernstein, Demonstration du théorème de Weierstrass fondéee sur le calcul de probabilities, Com. of the Kharkov Math. Soc., 13 (1912), 1-2.

[14] A. Boccuto, D. Candeloro: Integral and ideals in Riesz spaces, Inf. Sci., 179 (2009), 647-660.

[15] A. Boccuto, K. Demirci and S. Yildiz: Abstract Korovkin-type theorems in the filter setting with respect to relative uniform convergence, Turkish J. Math, 44 (2020), 1238-1249.

[16] A. Boccuto, X. Dimitriou: Convergence theorems for lattice group-valued measures, Bentham Science Publ., Sharjah, United Arab Emirates, (2015).

[17] A. Boccuto, X. Dimitriou: Korovkin-type theorems for abstract modular convergence, Results Math., 69 (2016), 477-495.

[18] H. Bohman, On approximation of continuous and of analytic functions, Arkiv Math., 2 (3) (1952), 43-56.

[19] J. Borsík, T. Šalát: On F-continuity of real functions, Tatra Mt. Math. Publ., 2 (1993), 37-42.

[20] P. L. Butzer, R. J. Nessel: Fourier Analysis and Approximation I, Academic Press, New York, London, (1971).

[21] E. Briem: Convergence of sequences of positive operators on $L^{p}$-spaces, in: Proc. of the Nineteenth Nordic Congress of Mathematicians,Reykjavik, (1984), 126-131, Visindafel. Isl., XLIV, Icel. Math. Soc. Reykjavik, 1985.

[22] K. Demirci: I-limit superior and limit inferior, Math. Commun., 6 (2) (2001), 165-172.

[23] K. Demirci, A. Boccuto, S. Yildiz and F. Dirik: Relative uniform convergence of a sequence of functions at a point and Korovkin-type approximation theorems, Positivity, 24 (2020), 1-11.

[24] K. Demirci, K. F. Dirik: A Korovkin-type approximation theorem for double sequences of positive linear operators of two variables in A-statistical sense, Bull. Korean Math. Soc., 47 (4) (2010), 825-837.

[25] R. A. DeVore, G. G. Lorentz: Constructive Approximation, Grund. Math. Wiss. 303, Springer Verlag, Berlin, Heidelberg, New York, (1993).

[26] K. Donner: Korovkin theorems in $L^{p}$-spaces, J. Funct. Anal., 42 (1) (1981), 12-28.

[27] O. Duman, M. Khan and C. Orhan: A-statistical convergence of approximation operators, Math. Ineq. Appl., 6 (4) (2003) 689-699.

[28] H. Fast: Sur la convergence statistique, Colloq. Math., 2 (1951), 241-244.

[29] A. D. Gadjiev: The convergence problem for a sequence of positive linear operators on unbounded sets, and theorems analogous to that of P.P. Korovkin, (Russian), Dokl. Akad. Nauk SSSR, 218 (5) (1974), 1001-1004.

[30] A. D. Gadjiev: Theorems of the type of P.P. Korovkin's Theorems, Mat. Zametki, 20 (5) (1976), 781-786.

[31] S. Karakuş, K. Demirci and O. Duman: Equi-statistical convergence of positive linear operators, J. Math. Anal. Appl., 339 (2008), 1065-1072.

[32] W. Kitto, D. E. Wulbert: Korovkin approximations in $L^{p}$-spaces, Pacific J. Math., 63 (1) (1976), 153-167.

[33] P. P. Korovkin: On convergence of linear positive operators in the spaces of continuous functions (Russian), Doklady Akad. Nauk. S.S.S.R., 90 (1953), 961-964.

[34] P. P. Korovkin: Linear operators and approximation theory, Hindustan, New Delhi, (1960).

[35] P. Kostyrko, T. Salat and W. Wilkzynski: I-convergence, Real Anal. Exchange, 26 (2) (2000/2001), 669-685. 
[36] W. M. Kozlowski: Modular Function Spaces, Pure Appl. Math., Marcel Dekker, New York, Basel, 1988.

[37] L. Maligranda: Korovkin theorem in symmetric spaces, Comment. Math. Prace Mat., 27 (1) (1987), 135-140.

[38] I. Mantellini: Generalized sampling operators in modular spaces, Comment. Math., 38 (1998), 77-92.

[39] H. I. Miller: A-statistical convergence of subsequence of double sequences, Boll. U.M.I. 8 (2007), 727-739.

[40] J. Musielak: Orlicz Spaces and Modular Spaces, Lecture Notes in Math., 1034, Springer-Verlag, Berlin, Heidelberg, New York, (1983).

[41] H. Nakano: Modulared Semi-Ordered Linear Spaces, Maruzen Co., Ltd, Tokyo, (1950).

[42] P. F. Renaud: A Korovkin theorem for abstract Lebesgue spaces, J. Approx. Theory, 102 (1) (2000), 13-20.

[43] P. M. Soardi: On quantitative Korovkin's theorem in multivariate Orlicz spaces, Math. Japonica, 48 (1998), 205-212.

[44] H. Steinhaus: Sur la convergence ordinaire et la convergence asymptotique, Colloq. Math., 2 (1951), 73-74.

\author{
CARLO BARDARO \\ UNIVERSITY OF PERUGIA \\ DEPARTMENT OF MATHEMATICS AND COMPUTER SCIENCE \\ 1, Via VANVITELLi, 06123 PERUGIA, ITALY \\ ORCID: 0000-0002-7567-4005 \\ E-mail address: carlo.bardaro@unipg.it \\ ANTONIO BOCCUTO \\ UNIVERSITY OF PERUGIA \\ Department of MATHEMATiCS ANd COMPUter SCiEnCE \\ 1, Via VANVITElli, 06123 PERUgia, ITAly \\ ORCID: 0000-0003-3795-8856 \\ E-mail address: antonio.boccuto@unipg.it \\ ILARIA MANTELLINI \\ UNIVERSITY OF PERUGIA \\ DEPARTMENT OF MATHEMATICS AND COMPUTER SCIENCE \\ 1, Via VANVITELli, 06123 PERUGIA, ITALY \\ ORCID: 0000-0002-3731-659X \\ E-mail address: ilaria . mantellini@unipg. it
}

